\title{
Trends in Personality Disorder Diagnosis in Hospitalized Patients: Analysis of a Ten-Year Time Series
}

\author{
Andrés Fontalba-Navas ${ }^{1^{*}}$, Luis Gutiérrez-Rojas ${ }^{2}$, Juan Pedro Arrebola ${ }^{3,4}$ and Jose Miguel Pena Andreu ${ }^{5}$ \\ ${ }^{1}$ Community Mental Health 1 Unit Huercal- Overa, Northern Area Health Management of Almeria, Andalusia Health, Spain \\ ${ }^{2}$ Group Research in Psychiatry and Neurosciences (STS -549), Institute of Neurosciences, University of Granada, Spain \\ ${ }^{3}$ Research Instituto Biosanitary IBS Granada, Hospital Universitario Virgen de las Nieves University of Granada, Spain \\ ${ }^{4}$ CIBER Epidemiology and Public Health (CIBERESP), Spain \\ ${ }^{5}$ Departamento of Psychiatry and Physiotherapy, University of Málaga, Spain
}

\begin{abstract}
Objectives: The aim of this study is to analyze the evolution of diagnoses of personality disorders and their subtypes.

Method: We studied the total population of patients diagnosed with personality disorder (PD) and admitted to acute psychiatric units in all general hospitals, a total of 19 hospitals in Andalusia (south Spain), between 1995 and 2004. The Andalusian Public Healthcare System provides services to universal coverage, so our results are representative of general population.

Results: We analyzed the use of each diagnosis in the clinical practice and discussed the observed change in their trends. During this period, there was an increase in borderline, narcissistic, avoidant and dependent PDs, while antisocial, schizoid, obsessive-compulsive and non-specific PDs remained stable and there was a decline in the numbers of histrionic, paranoid and schizotypal PDs.
\end{abstract}

Conclusion: We developed a statistical model reflecting the use of PD diagnoses in a clinical setting, a model that could be used to classify such diagnoses in the future.

Keywords: Borderline; Narcissistic; Dependent; Personality disorder; Diagnosis

\section{Introduction}

Personality disorders (PDs) are permanent, inflexible patterns of inner experience and behavior that deviates markedly from the expectations of the individual's culture. They begin in adolescence or early adulthood, remain stable over time and produce discomfort or prejudice to the subject [1].

These features, according to the International Classification of Diseases (ICD) [2], represent extreme or at least significant deviations from the way in which the normal individual within a culture perceives, thinks and feels and, above all, relates to others, or are evidently distinct from cultural expectations, as detailed in the Diagnostic and Statistical Manual of Mental Disorders (DSM-5) [1]. Under the latter classification, the deviation should be present in more than one of the following areas: cognitive, affective, impulse control, the need for gratification and relations with other persons.

PDs include lasting, deeply-rooted forms of behavior, which are manifested as stable patterns of response to a broad spectrum of individual and social situations, and encompass diverse aspects of psychological and behavioural functions. They are often accompanied by varying degrees of subjective stress and difficulties in social adjustment $[3,4]$.

Our aim in this study is to analyze the use of PD diagnoses in clinical practice and to discuss the changing trends observed. An analytical time-series study based on a 10-year linear regression was conducted to identify trends in the diagnostic use of PDs and their subtypes in a hospitalized population, and to reflect the changing use of international classifications during the study period.

This analysis is necessary to understand the trends of these disorders, so after we can implement strategies in their approach to the treatment.

This study highlights the real use made of each diagnosis in a large population, and reflects the validity of each construct applied.

\section{Methods}

\section{Study area and population}

We analyzed the change in diagnoses of PD and their subtypes in inpatients. Over a 10-year period. To do so, we studied the total population of patients diagnosed with PD and admitted to acute psychiatric units in general hospitals in Andalusia (southern Spain) between 1995 and 2004. The Andalusian Public Healthcare System provides services to universal coverage. Coverage was universal for residents in the territory in their right of access to universal health care by the recent Law 33/2011, General Public Health. Health services in Andalusia are free provision at the time of access, and are financed by general taxation.

In 2004, according to the Andalusian Institute of Statistics, the

*Corresponding author: Andrés Fontalba Navas, Community Mental Health 1Unit Huercal- Overa, Northern Area Health Management of Almería, Andalusia Health, Spain, Tel: 34 619674454; E-mail: andresfontalba@gmail.com

Received February 20, 2015; Accepted March 18, 2015; Published March 25 2015

Citation: Navas AF, Rojas LG, Arrebola JP, Andreu JMP (2015) Trends in Personality Disorder Diagnosis in Hospitalized Patients: Analysis of a TenYear Time Series. J Psychiatry 18: 271 doi:10.4172/2378-5756.1000271

Copyright: $\odot 2015$ Navas AF, et al. This is an open-access article distributed unde the terms of the Creative Commons Attribution License, which permits unrestricted use, distribution, and reproduction in any medium, provided the original author and source are credited 
region had a population of 7,975,672 inhabitants. Mental health care is provided by the Andalusian Health Service (SAS), a public healthcare agency that has 19 acute inpatient mental health units, which provide specialized services for both short and long-term hospitalization.

The data used for the analysis were extracted from the Minimum Basic Data Set (MBDS) for Andalusia. This is a hospital information collection system which includes a set of variables obtained when the patient is discharged, and are of an administrative, clinical and demographic nature. The MBDS is fully deployed in all hospitals in Andalusia, and has provided very reliable data since 1995. Therefore, the present study examines the period from the latter year until 2004, when information collection and purging was complete. Our research group requested the SAS Central Services to centralize the information obtained from all participating hospitals. The information thus compiled constituted a database of 99,444 individuals. The MBDS contains valuable information for understanding the health situation of a population, as well as collecting the usual demographic data (age, sex, place of residence), records the diagnosis that motivated admission (main diagnosis) factors risk comorbidities and complications that the patient during hospitalization (secondary diagnoses), some relevant diagnostic techniques and therapeutic interventions, (procedures). Finally, the MBDS comprises the date of admission and discharge of patients and their income circumstances (urgent, scheduled) and the fact that patient discharge (discharge to home, death, transfer to another hospital, etc...).

The inclusion criteria was to have a personality disorder diagnosis in the period.

The study protocol was approved by the ethic committee of Health Delegation Malaga.

\section{Statistical Analyses}

Time series analyses are widely used in with respect to economic statistics, but are not so common in epidemiology and medicine [5,6]. For the time series in question, we developed a linear regression model to predict the trends in percentage of hospitalized patients during the time sequence (1995-2004, 10 years).

The dependent variable was percentage of inpatients with a diagnosis of personality disorder, and the independent variable in the model of time series was time measured in years.

The shape of the relationships between the independent and dependent variables was visually evaluated using locally weighted scatterplot smoothing (LOWESS), a nonparametric local regression method. Diagnosis of the models was performed in order to ensure the goodness of fit and the fulfillment of implementation conditions. Generalized standard-error inflation factors were used to verify the absence of collinearity between independent variables, while homoscedasticity was tested by plotting residual against fitted values. The linearity of quantitative independent variables was checked with partial regression plots, and the normality of errors was verified by normal QQ plots with $95 \%$ confidence intervals.

\section{Results}

Figure 1 shows the time series model obtained from our analysis of 10 years of hospital admissions by patients with at least one diagnosis of PD. This model illustrates the growth in cases of $\mathrm{PD}$ with respect to total diagnoses. The trend is a rising one $(\mathrm{x}=0.86)$, and this linear model is statistically significant $(\mathrm{R}=0.918, \mathrm{p}=0.000)$.
The estimated growth of each subtype of PD diagnosis is classified as shown in Table 1, assuming the constant of the trend line is positive, equal to 0 (no growth, no statistical significance) or negative.

\section{Discussion}

Our analysis reveals an increase in borderline, narcissistic, avoidant and dependent PDs in the study area. Patients diagnosed with borderline $\mathrm{PD}$, hospitalized and treated in acute units, are associated with the occurrence of major adverse effects, including suicide, real or attempted, and the camouflaging of risk behavior. This outcome could be explained by low diagnostic sensitivity to this PD subtype, as reported elsewhere [7]. Our results are similar to other epidemiologic studies in hospital settings [8]. They concluded with their prevalence that borderline PD is associated with more frequent admissions and numerous management challenges, suggesting the need for comprehensive screening, coordination of care, and specialized treatment programming.

Dependent PDs is frequent in individuals who have a generalized and excessive desire to be taken care of (submissive or clinging

\begin{tabular}{|l|c|c|c|c|}
\hline & Trend & Constant & $\begin{array}{c}\text { Standard } \\
\text { error }\end{array}$ & p \\
\hline POSITIVE GROWTH & & & & \\
\hline Borderline & 1.17 & 13,7 & 0.25 & 0.02 \\
\hline Dependent & 0.19 & 2.4 & 0.41 & 0.05 \\
\hline Anxious-Avoidant & 0.13 & -0.08 & 0.09 & 0.01 \\
\hline Narcissistic & 0.09 & 0.5 & 0.41 & 0.05 \\
\hline STABLE & & & & \\
\hline Non-specific & 0.03 & 35 & 0.28 & 0.90 \\
\hline Antisocial & 0.04 & 2.7 & 0.18 & 0.80 \\
\hline Schizoid & 0.06 & 2.6 & 0.08 & 0.51 \\
\hline Obsessive-Compulsive & -0.21 & 2.5 & 0.05 & 0,73 \\
\hline NEGATIVE GROWTH & & & & \\
\hline Paranoid & -0.33 & 23.34 & 0.1 & 0.01 \\
\hline Histrionic & -0.25 & 8.55 & 0.24 & 0.03 \\
\hline Schizotypal Trend: Beta coefficient & -0.10 & 2.54 & 0.06 & 0.01 \\
\hline in linear regression model & & & & \\
\hline
\end{tabular}

Table 1: Linear regression models of $P D$ diagnosis trends in the time seriesTrends in percentage of inpatients with diagnosis of PD during 10 years.

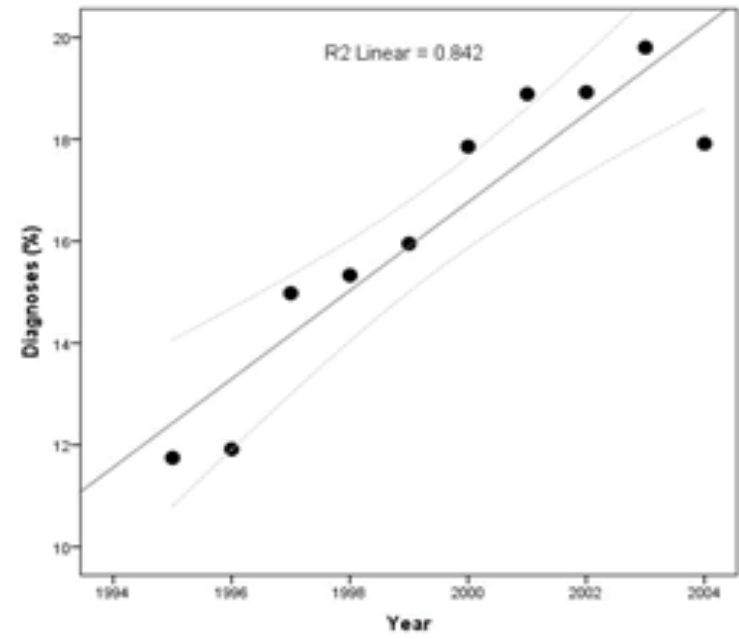

Figure 1: Observed trend in ten-year time series of the diagnosis of personality disorders in a hospitalised population 
behaviour) and a great fear of separation. These features are subject to strong cultural and social influences [9], and therefore environmental factors could underlie their increasing presence.

The main feature of anxious-avoidant PD is a general pattern of social inhibition, feelings of inadequacy and hypersensitivity to negative evaluation. This PD subtype has also been associated with the diagnosis of social phobia, which has also acquired greater prominence in recent years [10]. Narcissistic PD is relatively uncommon, although its presence is increasing. The overlap in diagnostic criteria with other Category B disorders [11] could explain this trend. With respect to the second group of PDs, our analysis indicates that diagnoses of antisocial, schizoid, obsessive-compulsive and non-specific PDs presented a stable long-term trend over the study period.

The two major classification systems, DSM and ICD, apply different criteria in defining antisocial PD [12]. Nevertheless, this diagnosis presents a stable long-term trend.

Schizoid disorder is closely associated with generalized developmental disorders and some authors have even located it within the area of autism. The chronicity and long-term stability of these disorders may correspond to the time series observed in this study [13]. With respect to obsessive-compulsive PDs, DSM- 5 and ICD- 10 broadly define the same disorder, but in the latter case it is termed anankastic PD. Similarly to antisocial PD, the time series reflects a linear trend and a pattern that, despite inter-annual variations, is relatively stable.

The category of non-specific PD is the final diagnostic category, which is used when a PD is believed to be present but there is insufficient information to allow a more specific diagnosis to be made. However, this diagnosis is associated with a worse prognosis than the categories in which the subtype is specified [14]. In the evolution of diagnoses, paradoxically but in agreement with previous findings, this diagnostic category remains stable over time.

The third group of diagnostic categories are those which have experienced negative growth, namely histrionic, paranoid and schizotypal PDs. Thus, the presence of histrionic PDs decreased during the time series examined; furthermore, the psychometric construction of this diagnosis has recently been questioned [15]. These results corroborate our prior hypothesis that the category of histrionic PD is tending to be combined with that of borderline PD [16].

In recent years, relatively little interest has been shown in the study of paranoid $\mathrm{PD}$, which could be related to the decreasing presence of this diagnosis. ICD-10 does not consider schizotypal PD to be a true $\mathrm{PD}$, and includes it in the category termed schizophrenia and schizotypal and delusional disorders. In our study, the number of patients diagnosed with schizotypal PD tended to decrease, with a possible transfer to other categories of chronic psychosis.

In the study design applied, some patients were diagnosed with PD without taking into account other or overlapping diagnostic categories. However, this approach allowed us to examine each subtype separately and to measure the trend for each diagnostic category. This results suggest the importance of stability in the diagnostic criteria for PD [17]. Changes in future classifications will affect to these trends. This is the first study in mental health epidemiology developed by a Heath Care System with a large sample followed during 10 years. Due to universal coverage of Andalusian Health System, results are comparable to general population.

This study reflects the real use in a clinical setting of each PD diagnosis. The analysis of trends in diagnosis could be used to design future diagnostic classifications. Moreover, we highlight the difference between the inclusion of certain categories in theoretical classifications and their utility in real clinical practice.

\section{References}

1. Diagnostic and statistical manual of mental disorders (5thed.) Arlington, American Psychiatric Association 2013.

2. CIE-10, Décima revisión de la clasificación internacional de las enfermedades. Trastornos mentales y del comportamiento. Meditor, Madrid, 1992

3. Widiger TA, Samuel DB (2005) Evidence-based assessment of personality disorders. Psychol Assess 17: 278-87.

4. Siever LJ (2005) Endophenotypes in the personality disorders. Dialogues Clin Neurosci 7: 139-51.

5. Rios M, Garcia JM, Cubedo M, Pérez D (1996) Análisis de series temporales en la epidemiología de la fiebre tifoidea. Med Clin 106: 686-689.

6. Kis M (2002) Analysis of the time series for some causes of death. Stud Health Technol Inform 90: 439-443.

7. Comtois KA, Carmel A (2014) Borderline personality disorder and high utilization of inpatient psychiatric hospitalization: concordance between research and clinical diagnosis. J Behav Health Serv Res. May 30.

8. Leontieva L, Gregory R (2013) Characteristics of patients with borderline personality disorder in a state psychiatric hospital. J Pers Disord 27: 222-32.

9. Chen Y, Nettles ME, Chen SW (2009) Rethinking dependent personality disorder: comparing different human relatedness in cultural contexts. J Nerv Ment Dis 197: 793-800.

10. Reich J (2009) Avoidant personality disorder and its relationship to socia phobia. Curr Psychiatry Rep 11: 89-93.

11. Cain NM, Pincus AL, Ansell EB (2008) Narcissism at the crossroads: phenotypic description of pathological narcissism across clinical theory, social/personality psychology, and psychiatric diagnosis. Clin Psychol Rev 28: 638-56.

12. Ottosson H, Ekselius L, Grann M, Kullgren G (2002) Cross-system concordance of personality disorder diagnoses of DSM-IV and diagnostic criteria for research of ICD-10. J Pers Disord 16: 283-292.

13. Kosson DS, Blackburn R, Byrnes KA, Park S, Logan C, et al. (2008) Assessing interpersonal aspects of schizoid personality disorder: preliminary validation studies. J Pers Assess 90: 185-189.

14. Johnson JG, First MB, Cohen P, Skodol AE, Kasen S, et al. (2005) Adverse outcomes associated with personality disorder not otherwise specified in a community sample. Am J Psychiatry 162: 1926-1932.

15. Blagov PS, Westen D (2008) Questioning the coherence of histrionic personality disorder: borderline and hysterical personality subtypes in adults and adolescents. J Nerv Ment Dis196: 785-797.

16. Pena-Andreu JM, Fontalba A, Carrera M, Martinez JL, Rodriguez MI (2005) Refining the personality disorder diagnosis. Am J Psychiatry 162: 1030-1031.

17. Orts Clemmensen LM, Olrik Wallenstein Jensen S, Zanarini MC, Skadhede $S$, Munk-Jorgensen $P(2013)$ Changes in treated incidence of borderline personality disorder in denmark: 1970-2009. Can J Psychiatry 58: 522-528. 